\title{
Diagnosis and Treatment of Joint Fluid Accumulation After Total Knee Arthroplasty in Rheumatoid Arthrosis Patients: A Report of Four Patients
}

\author{
Hiroyuki Tsuchie ${ }^{a, b, d}$, Seiya Miyamoto a, b, Seietsu Senma ${ }^{a}$, Yuichiro Narita ${ }^{a}$, Yuji Hatakeyama ${ }^{a}$, \\ Kana Sasakia ${ }^{a}$ Yoichi Shimada ${ }^{c}$
}

\begin{abstract}
Joint fluid after total knee arthroplasty (TKA) has various causes, and the specific cause must be identified. Even if joint fluid occurs in rheumatoid arthrosis (RA) patients after TKA, other causes still have to be ruled out. We describe four cases of joint fluid after TKA in RA patients, in a total of 34 consecutive patients (50 knees), and review the literature. When a definitive diagnosis or treatment of joint fluid is difficult, synovectomy by arthroscopy may be effective both for the diagnosis and treatment.
\end{abstract}

Keywords: Joint fluid; Rheumatoid arthrosis; Synovectomy; Total knee arthroplasty

\section{Introduction}

Joint fluid after total knee arthroplasty (TKA) has various causes, and the specific cause must be identified. Even if joint fluid occurs in rheumatoid arthrosis (RA) patients after TKA, we have to distinguish other causes, such as infection, excluding an increase in RA activity. Because there have been only a few systematic reports of joint fluid after TKA in RA patients, the causes and frequency have not been clearly established.

We herein describe four cases of joint fluid after TKA in RA patients, and review the literature.

\section{Case Reports}

A total of 34 consecutive patients (50 knees) with RA receiv-

\footnotetext{
Manuscript accepted for publication January 29, 2016

aDivision of Orthopedic Surgery, Nakadori General Hospital, 3-15, Misonocho, Minami-dori, Akita 010-8577, Japan

bAkita Orthopedic Group on Rheumatoid Arthritis (AORA), Akita, Japan

'Department of Orthopedic Surgery, Akita University Graduate School of Medicine, 1-1-1 Hondo, Akita 010-8543, Japan

${ }^{\mathrm{d} C}$ Corresponding Author: Hiroyuki Tsuchie, Department of Orthopedic Surgery, Nakadori General Hospital, 3-15, Misono-cho, Minami-dori, Akita 0108577, Japan. Email: tuchikiti@yahoo.co.jp
}

doi: http://dx.doi.org/10.14740/jmc2429w ing TKA who visited a regional center hospital in Akita, Japan between April 2002 and March 2012 were investigated in this study. There were four males and 30 females, with a mean age at the time of surgery of 67.3 years ( $47-80$ years). The mean postoperative observation period was 6.6 years ( $3-12$ years).

In these patients, there were four patients (four knees) with knee joint fluid more than 1 year after surgery. The ages of the four patients at the time of joint fluid ranged from 52 to 74 years, with a mean of 67.3 years, and all patients were female. The period up to joint fluid onset after surgery ranged from 14 to 63 months, with a mean of 39.5 months.

\section{Case 1}

A 74-year-old woman presented to our outpatient clinic with joint fluid of the left knee with a year and 4-month history. She had been treated for RA in another hospital for 34 years, and had received left TKA surgery in our hospital 2 years and 6 months ago. She had been prescribed methotrexate (MTX) for RA treatment. Although her left knee showed joint fluid, redness and heart sensation were not present. On laboratory

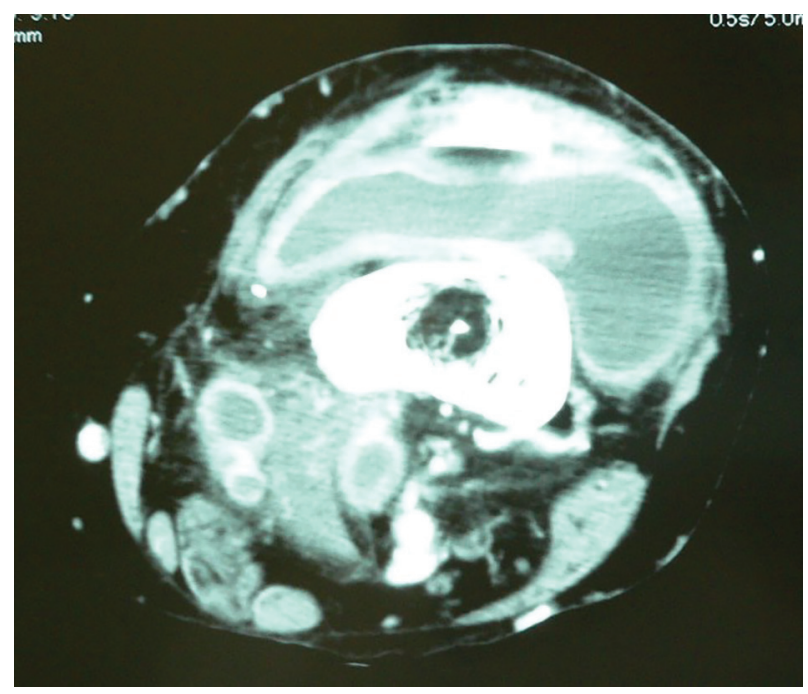

Figure 1. Enhanced computed tomography (CT) of the left knee joint at the initial visit (axial section). CT showed the accumulation of joint fluid and a contrasted, thickened synovium. 


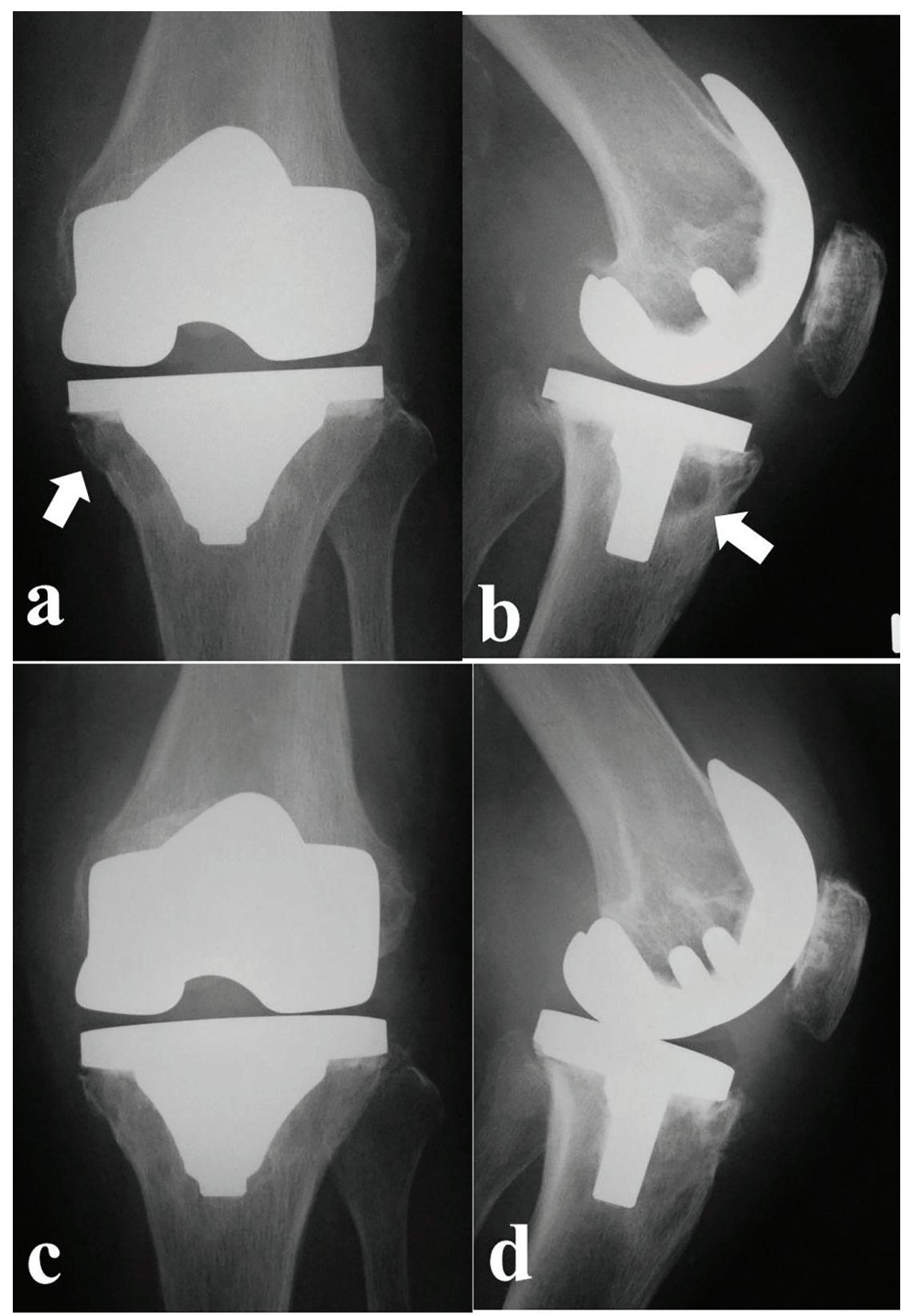

Figure 2. Antero-posterior and lateral radiographs of the left knee joint at 1 year and 2 months after the initial visit (a, b), and 3 months postoperatively $(\mathrm{c}, \mathrm{d})$. Although plain radiographs initially showed erosion around the tibial implant (arrows) (a,b), it disappeared 3 months postoperatively (b).

examinations, C-reactive protein (CRP) and matrix metalloproteinase-3 (MMP-3) of the plasma were high (CRP: 10.4 $\mathrm{mg} / \mathrm{dL}, \mathrm{MMP}-3: 265 \mathrm{ng} / \mathrm{mL})$. Enhanced computed tomography (CT) showed the accumulation of joint fluid and a contrasted, thickened synovium (Fig. 1). Because bacteria were not detected in the joint fluid, we thought that reservoir of joint fluid was caused by increasing RA activity, and prescribed etanercept.

Although inflammation decreased, it exacerbated again 1 year and 2 months after the initial visit, and we prescribed tocilizumab. Although inflammation was again decreased (CRP:
$0.1 \mathrm{mg} / \mathrm{dL}, \mathrm{MMP}-3: 220 \mathrm{ng} / \mathrm{mL}$ ), joint fluid of the left knee was continuously present. In addition, erosion around the tibial implant was seen on plain radiographs (Fig. 2a). We performed arthroscopic synovectomy of the left knee 1 year and 4 months after the initial visit. White and red synovial tissue filled the joint (Fig. 3). Histopathologic examination confirmed the diagnosis of RA, and fibrin deposits, a synovium with degenerative necrosis, hyperplastic capillaries, and lymphocyte infiltration were observed. There was no evidence of bacteria (Fig. 4). At 3 months postoperatively, inflammation was decreased (CRP: $0.0 \mathrm{mg} / \mathrm{dL}, \mathrm{MMP}-3: 144 \mathrm{ng} / \mathrm{mL}$ ), and erosion around the tibial 


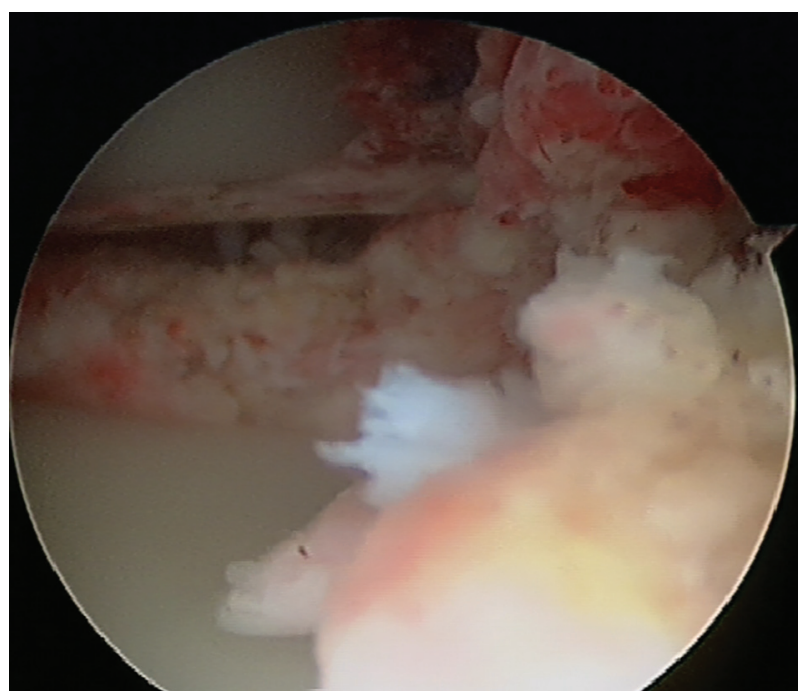

Figure 3. Arthroscopy of the left knee showed that white and red synovium filled the joint.

implant disappeared on plain radiographs (Fig. 2b). Joint fluid then disappeared, and she did not have any joint fluid in the left knee at the most recent follow-up at 3 years postoperatively.

\section{Case 2}

A 74-year-old woman presented with joint fluid of the right knee 5 years and 3 months after right TKA. She had been treated for RA for 9 years. She had been prescribed MTX and etanercept for RA treatment. Although her right knee showed joint fluid, redness and a local heart were not shown. Laboratory examinations showed marked inflammation (CRP: 3.1 $\mathrm{mg} / \mathrm{dL}$, MMP-3: $332 \mathrm{ng} / \mathrm{mL}$ ), but bacteria were not detected in the joint fluid. Enhanced CT showed the accumulation of joint fluid and a contrasted, thickened synovium. We adjusted the medication, and inflammation decreased. Although joint fluid accumulation did not improve, we observed her because she reported the absence of pain.

She complained of pain and a local heat of the right knee 2 years and 6 months after the initial visit. Although inflammatory reaction increased again (CRP: $5.4 \mathrm{mg} / \mathrm{dL}$, MMP-3: $283 \mathrm{ng} / \mathrm{mL}$ ), bacteria were again not detected in the joint fluid. We performed arthroscopic synovectomy of the right knee 2 years and 9 months after the initial visit. White synovial tissue mainly filled the joint. Histopathologic examination confirmed the diagnosis of RA, and there was no evidence of bacteria. At 2 months postoperatively, inflammation was decreased (CRP: $0.0 \mathrm{mg} / \mathrm{dL}$, MMP-3: $90.6 \mathrm{ng} / \mathrm{mL}$ ), and joint fluid disappeared. She did not show any joint fluid accumulation at the most recent follow-up of 2 years postoperatively.

\section{Case 3}

A 69-year-old woman presented with joint fluid of the left knee 4 years and 2 months after right TKA. She had been treated

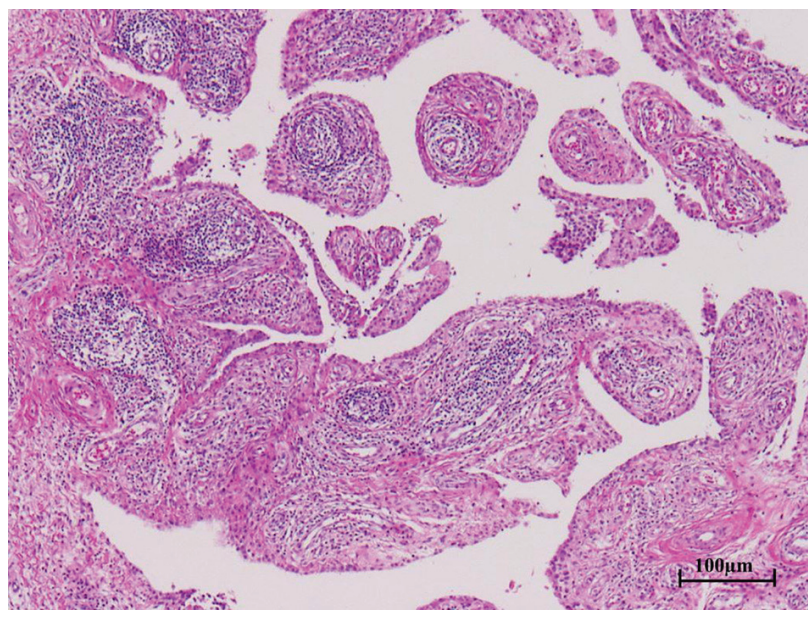

Figure 4. Histopathological findings on Villanueva bone staining. Original magnification $\times 40$. Fibrin deposits, a synovium with degenerative necrosis, hyperplastic capillaries, and lymphocyte infiltration were observed. There was no evidence of bacteria.

for RA for 14 years. She had been prescribed MTX, steroid, and etanercept for RA treatment. Although she had pain of the left knee, redness and a local heart were not shown. Laboratory examinations showed marked inflammation (CRP: 12.5 $\mathrm{mg} / \mathrm{dL}$, MMP-3: $231.9 \mathrm{ng} / \mathrm{mL}$ ), but bacteria were not detected in the joint fluid. We increased the amount of etanercept, and inflammation was decreased (CRP: $0.7 \mathrm{mg} / \mathrm{dL}, \mathrm{MMP}-3: 141.9$ $\mathrm{ng} / \mathrm{mL}$ ) only 2 months after the initial visit. Joint fluid and pain disappeared, and she did not show any joint fluid accumulation at the most recent follow-up at 1 year and 4 months after the initial visit.

\section{Case 4}

A 52-year-old woman presented with joint fluid of the right knee 2 years and 3 months after right TKA. She had been treated for RA for 3 years. She had been prescribed MTX, steroid, tacrolimus, and tocilizumab for RA treatment. Although she had mild pain of the right knee, redness and a local heart were not shown. Laboratory examinations showed no inflammation (CRP: $0.0 \mathrm{mg} / \mathrm{dL}$, MMP-3: $40.3 \mathrm{ng} / \mathrm{mL}$ ), and bacteria were not detected in the joint fluid. Because of only mild pain of the right knee and the absence of inflammation, we observed her and continued the administration of medicine until the most recent follow-up at 2 years after the initial visit.

\section{Discussion}

Although both the amount of joint fluid and protein in the fluid increase due to surgical invasion until 3 months after TKA, these decrease gradually 3 months later [1]. So, we can consider that there are causes other than surgical invasion if joint fluid occurs at 3 months or more after surgery. As causes of joint fluid after TKA, we can classify them into five different groups: deep infection (DI), temporarily increased activity of 
RA (IRA), wear particle-induced synovitis (PS), metal sensitivity to implant metals (MS), and nonspecific synovitis (NS) [2-4]. Because DI, PS, and MS can not be treated conservatively, it is important to clarify their causes. Although we may easily consider IRA as the cause of joint fluid in RA patients, joint disorder may deteriorate when we use some RA medicines for DI patients. Although there is a report of a differential test using fluorescence-activated cell sorter [5], various examination results, such as a bacterial culture test, detection of wear debris, and the RA activity index, are needed for a confident diagnosis. Although a bacterial culture test for joint fluid is important, DI can not be completely ruled out even if bacteria are not detected by this examination [6]. If the cause is not clear, it is possible to diagnose more accurately by ablating the synovium as in the present cases.

If the cause of joint fluid is IRA, it is important to treat it using medicines for RA, such as biologics, to control RA activity. In the present case 3 , joint fluid decreased only on treatment with drugs. Although pathological examination and a bacterial culture test of the synovium are needed to fully rule out infection, actually, we often initially start medical treatment to control RA activity if we consider IRA based on some examinations, even if we do not carry out synovium examinations. However, when it is difficult to decrease joint fluid, we should conduct synovectomy [7]. In the present cases, surgical treatment by arthroscopy brought about favorable results, and this can be expected to facilitate a reliable diagnosis and marked therapeutic effect.

Niki et al reported that the incidence of joint fluid after TKA was less than 3\% [4]. In their data, the incidence was high $(8 \%)$, although the study involved a small number of cases. In RA patients only, the incidence of joint fluid after TKA may have been high because of IRA. In addition, they investigated joint fluid in patients after TKA whose original diagnosis was osteoarthritis (OA) or RA, and the most frequent cause of post TKA effusion was IRA in RA, and NS in OA [4]. Although the cause of joint fluid in patients was mostly IRA in the present cases, we need to perform further studies with a larger number of cases.

In conclusion, we present four cases of joint fluid after
TKA in RA patients showing some courses on synovectomy or prescribing RA medicines. When a definitive diagnosis or treatment of joint fluid is difficult, synovectomy by arthroscopy may be effective both for the diagnosis and treatment.

\section{Conflict of Interest}

We do not have any conflict of interest.

\section{References}

1. Delecrin J, Oka M, Takahashi S, Yamamuro T, Nakamura T. Changes in joint fluid after total arthroplasty. A quantitative study on the rabbit knee joint. Clin Orthop Relat Res. 1994;307:240-249.

2. Hallab NJ, Meritt K, Jacobs JJ. Current concept review; metal sensitivity in patients with orthopedic implants. J Bone Joint Surg Am. 2001;83:428-436.

3. Freedmont AJ, Denton J, Chuck A, Holt PJ, Davies M. Diagnostic value of synovial fluid microscopy: A reassessment and rationalization. Ann Rheum Dis. 1991;50:101107.

4. Niki Y, Matsumoto H, Otani T, Tomatsu T, Toyama Y. Five types of inflammatory arthritis following total knee arthroplasty. J Biomed Mater Res A. 2007;81(4):10051010 .

5. Niki Y, Matsumoto H, Otani T, Yatabe T, Funayama A, Maeno S, Tomatsu T, et al. Phenotypic characteristics of joint fluid cells from patients with continuous joint effusion after total knee arthroplasty. Biomaterials. 2006;27(8):1558-1565.

6. Erdem H, Baylan O, Simsek I, Dinc A, Pay S, Kocaoglu M. Delayed diagnosis of tuberculous arthritis. Jpn J Infect Dis. 2005;58(6):373-375.

7. Ogawa H, Itokazu M, Ito Y, Fukuta M, Shimizu K. The therapeutic outcome of minimally invasive synovectomy assisted with arthroscopy in the rheumatoid knee. Mod Rheumatol. 2006;16(6):360-363. 